\title{
Assessment of Distress
}

National Cancer Institute

\section{Source}

National Cancer Institute. Assessment of Distress. NCI Thesaurus. Code C137856.

The act of determining, usually through interviews and observation, the status of an individual's distress. 